ORIGINAL ARTICLE

\title{
Blockade of interleukin- 6 receptor in the periphery promotes rapid and sustained antidepressant actions: a possible role of gut-microbiota-brain axis
}

\author{
J-c Zhang, W Yao, C Dong, C Yang, Q Ren, M Ma and K Hashimoto
}

\begin{abstract}
Depression is a common, severe and chronic psychiatric disease. Although the currently available antidepressants have been used in the treatment of depression, their beneficial effects are limited. Accumulating evidence suggests that pro-inflammatory cytokines such as interleukin-6 (IL-6) have an important role in the pathogenesis of depression. This study was undertaken to examine whether anti-mouse IL-6 receptor antibody (MR16-1) induces antidepressant effects in a social defeat stress model. Intravenous injection of MR16-1 induced rapid-onset and long-lasting antidepressant effects in susceptible mice after social defeat stress through its anti-inflammatory actions. In contrast, intracerebroventricular injection of MR16-1 induced no antidepressant effects in susceptible mice. Furthermore, treatment with MR16-1 could significantly normalize alterations in the expression of synaptic proteins (postsynaptic density protein 95 and a-amino-3-hydroxy-5-methyl-4-isoxazolepropionic acid receptor 1) and dendritic spine density in the brain regions of susceptible mice. Gut microbiota analysis using $16 \mathrm{~S}$ ribosomal RNA gene sequencing showed that MR16-1 significantly improved decreased Firmicutes/Bacteroidetes ratio in susceptible mice. It also significantly improved decreased levels of Oscillospira in susceptible mice. These findings suggest that peripheral IL- 6 has a key role in the pathogenesis of depression and that the blockade of IL-6 receptor in the periphery might have rapid-onset and long-lasting antidepressant effects by normalizing the altered composition of gut microbiota in susceptible mice after social defeat stress. Therefore, the blockade of IL-6 receptor in the periphery shows promise as a novel therapeutic approach for depressed patients with higher IL-6 blood levels.
\end{abstract}

Translational Psychiatry (2017) 7, e1138; doi:10.1038/tp.2017.112; published online 30 May 2017

\section{INTRODUCTION}

Depression is a significant contributor to the global burden of disease and affects people in all communities across the world. It is estimated to affect 350 million people, and almost 1 million lives are lost annually due to suicide. The World Health Organization predicts that depression will be the single leading cause of burden of all health conditions by $2030 .^{1}$ Although inflammation has a central role in the pathogenesis of depression, ${ }^{2-7}$ the precise mechanisms underlying inflammation-induced depression remain undetermined. Accumulating evidence suggests that interleukin- 6 (IL-6), one of the pro-inflammatory cytokines, has an important role in the pathogenesis of depression. ${ }^{8-14}$ Meta-analyses showed that depressed patients have higher levels of multiple inflammatory markers, including IL-6. ${ }^{15-17}$ Furthermore, blood levels of IL-6 in patients with suicidality were significantly higher than those in patients without suicidality and healthy control subjects, suggesting that peripheral IL-6 may be associated with suicidal ideation, a core symptom of depression. ${ }^{11}$ Interestingly, we reported that alterations in peripheral, but not brain, IL-6 level might contribute to resilience versus susceptibility to inescapable stress in the rat learned helplessness model. ${ }^{18}$ Taken together, it is likely that peripheral IL- 6 might be involved in the pathogenesis of inflammation-induced depression. ${ }^{12,13,18}$

The gut-microbiota-brain axis is a complex multi-organ bidirectional signaling system between the microbiota and brain that has a fundamental role in host physiology, homeostasis, development and metabolism. ${ }^{19-21}$ Accumulating evidence suggests the reproducible and consistent effects of microbial states on mouse behavior, supporting the role of microbiota in behavior. ${ }^{22-24}$ Recent studies have demonstrated that abnormalities in the composition of gut microbiota might have a causative role in depression. ${ }^{25-27}$

Considering the key role of IL- 6 in depression, the present study investigated whether anti-mouse IL-6 receptor antibody (MR16-1) induces antidepressant-like effects in the social defeat stress model. Next, we examined the role of synaptogenesis in the selected brain regions for the antidepressant actions of MR16-1. Finally, we examined the role of gut microbiota in the antidepressant actions of MR16-1 in the social defeat stress model.

\section{MATERIALS AND METHODS}

\section{Animals}

Male adult C57BL/6 mice $(n=265)$, aged 8 weeks (body weight $20-25 \mathrm{~g}$, Japan SLC, Hamamatsu, Japan), and male CD1 mice $(n=40)$, aged 14 weeks (body weight $40-45 \mathrm{~g}$, Japan SLC) were used in the experiments. The animals were housed under controlled temperature and $12 \mathrm{~h}$ light/ dark cycles (lights on between 0700 and $1900 \mathrm{~h}$ ), with ad libitum food and water. The protocol was approved by the Chiba University Institutional Animal Care and Use Committee (permission number: 27-125 and 28-273). 
a

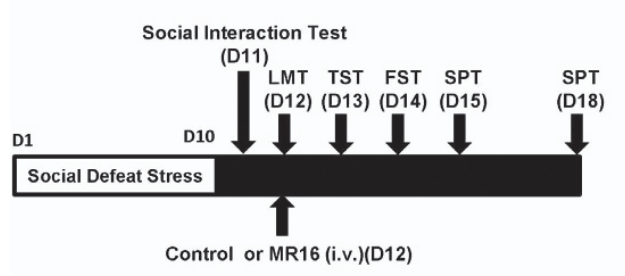

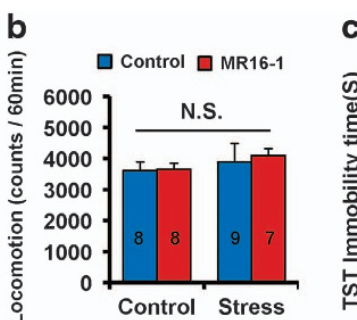

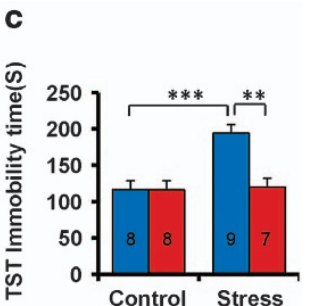

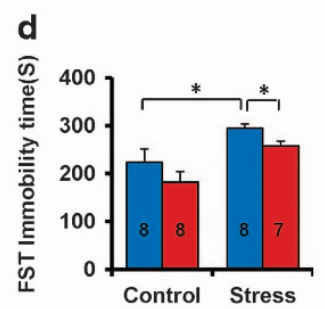
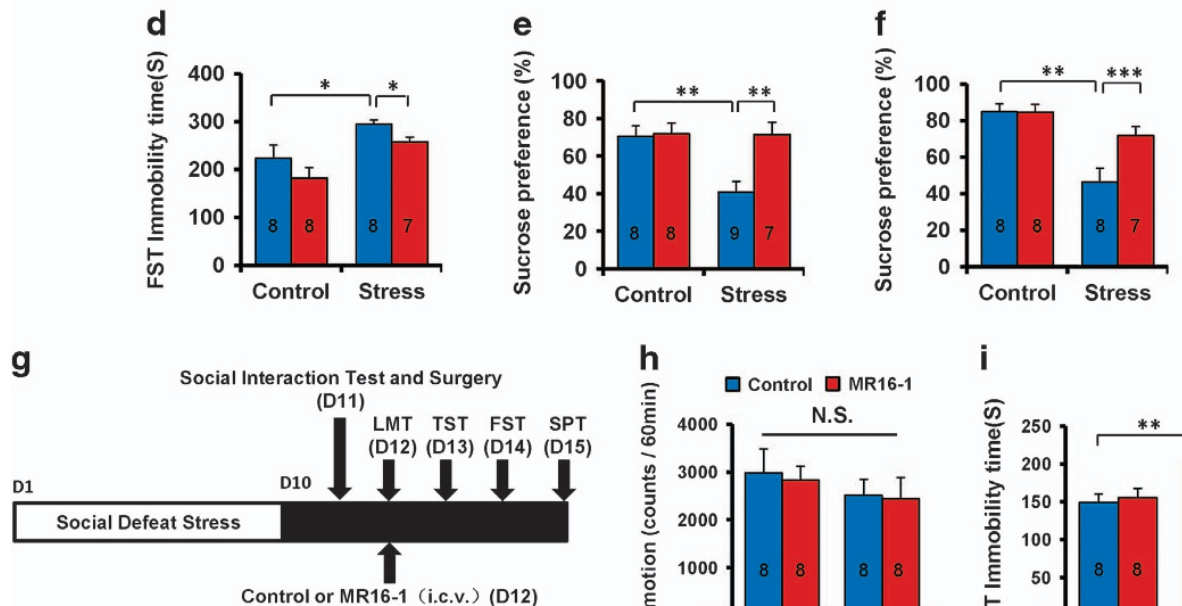

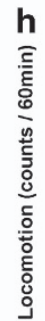
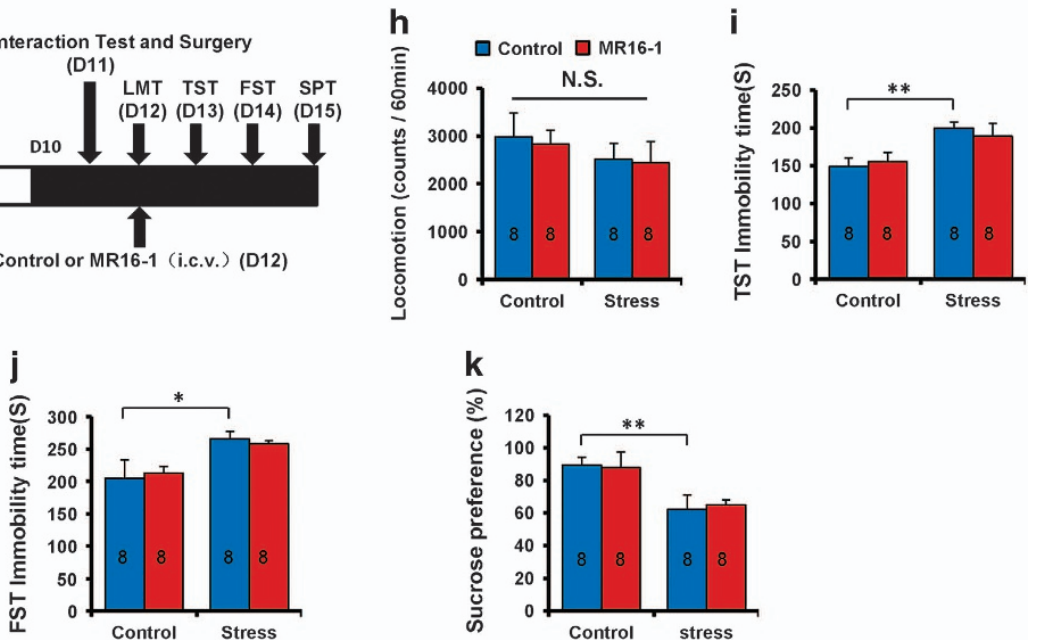

Figure 1. Antidepressant effects of MR16-1 in social defeat stress model. (a) Schedule of social defeat stress (10 days), social interaction test, drug treatment (intravenous (i.v.)), and behavioral tests. (b) Locomotion test (LMT). (c) Tail suspension test (TST). (d) Forced swimming test (FST). (e) One \% sucrose preference test (SPT). (f) One \% SPT. Two-way analysis of variance (ANOVA) revealed the results (LMT (b), Stress: $\mathrm{F}_{1,31}=0.843, P=0.366 ; \mathrm{MR} 16-1: \mathrm{F}_{1,31}=0.064, P=0.801$; interaction: $\left.\mathrm{F}_{1,31}=0.078, P=0.782\right)$, (TST (c), Stress: $\mathrm{F}_{1,31}=11.351, P=0.002 ; \mathrm{MR} 16-1$ : $F_{1,31}=9.425, P=0.005$; interaction: $\left.F_{1,31}=9.317, P=0.005\right)$, (FST (d); Stress: $F_{1,30}=15.389, P=0.001 ;$ MR16-1: $F_{1,30}=4.363, P=0.046$; interaction: $\left.\mathrm{F}_{1,27}=0.008, P=0.929\right),\left(\mathrm{SPT}(\mathbf{e}) ;\right.$ Stress: $\mathrm{F}_{1,31}=6.312, P=0.018 ; \mathrm{MR} 16-1: \mathrm{F}_{1,31}=7.259, P=0.012$; interaction: $\left.\mathrm{F}_{1,31}=6.002, P=0.021\right),(\mathrm{SPT}(\mathbf{f})$; Stress: $F_{1,30}=5.126, P=0.032 ; M R 16-1: F_{1,30}=5.086, P=0.032$; interaction: $\left.F_{1,30}=20.956, P<0.001\right)$. The values represent the mean \pm s.e.m. $(n=7-9) .{ }^{*} P<0.05,{ }^{* *} P<0.01,{ }^{* * *} P<0.001$ compared with the control-treated stress group. (g) Schedule of social defeat stress (10 days), social interaction test and surgery, drug treatment (intracerebroventricular (i.c.v.)), and behavioral tests. (h) LMT. (i) TST. (j) FST. (k) One \% SPT. Two-way ANOVA revealed the results $\left(\operatorname{LMT}(\mathbf{h})\right.$, Stress: $F_{1,32}=1.406, P=0.245 ;$ MR16-1: $F_{1,32}=0.091, P=0.765$; interaction: $F_{1,32}=0.009$,

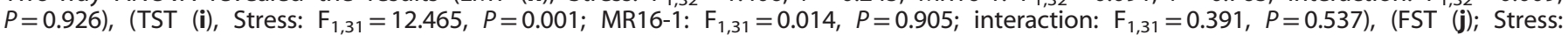
$F_{1,31}=12.149, P=0.002 ; M R 16-1: F_{1,31}=0.027, P=0.871$; interaction: $\left.F_{1,31}=0.476, P=0.496\right)$, (SPT (k), Stress: $F_{1,31}=12.737, P=0.001 ; M R 16-1$ : $\mathrm{F}_{1,31}=0.008, P=0.929$; interaction: $\left.\mathrm{F}_{1,31}=0.094, P=0.762\right)$. The values represent the mean \pm s.e.m. $(n=8)$. ${ }^{*} P<0.05$ and ${ }^{* *} P<0.01$ compared with the control+stressed group. The number in the column is the number of mice. NS, not significant.

Intravenous administration of rat MR16-1

Rat MR16-1 (a gift from Chugai Pharmaceutical (Tokyo, Japan)) was used. ${ }^{28}$ On the injection day, the mice were given intravenous injection of MR16-1 ( $2 \mathrm{mg}$ per $0.2 \mathrm{ml}$ per mouse) or rat lgG isotype control (clone $43414, \mathrm{R} \& \mathrm{D}$ Systems, Minneapolis, MN, USA).

Intracerebroventricular administration of MR16-1

After the social interaction test (day 11), susceptible mice were anesthetized with isoflurane and pentobarbital $\left(5 \mathrm{mg} \mathrm{ml}^{-1}\right.$ and $0.15 \mathrm{ml}$ per mouse) and placed in a stereotaxic frame. The cannulas were placed into the lateral ventricles $(+0.02$ anteroposterior, +1.0 mediolateral,-1.5 dorsoventral). ${ }^{29}$ Twenty-four hours after surgery (day 12 in Figure 1g), MR16-1 (0.01 mg $\mathrm{ml}^{-1}, 0.1 \mu \mathrm{min}^{-1}$ for $20 \mathrm{~min}$ ) or control lgG was injected into susceptible mice.

\section{Social defeat stress model}

Briefly, C57BL/6 mice were exposed to a different CD1 aggressor mouse each day for $10 \mathrm{~min}$ for 10 days. ${ }^{30-39}$ After the social defeat session, the resident $C D 1$ mouse and intruder mouse were housed in one-half of the cage separated by a perforated Plexiglas divider to allow visual, olfactory and auditory contact for the remainder of the $24 \mathrm{~h}$ period. Twenty-four hours after the last session, all the mice were housed individually. On day 11 , a social avoidance test was performed to identify the subgroups of mice that were susceptible and unsusceptible to social defeat stress. Approximately $70 \%$ of mice were susceptible in this study. Only susceptible mice were used in the subsequent experiments.

A social interaction test was performed 1 day (day 11$)$ after the last social defeat session. For this test, an open-field arena $(42 \times 42 \mathrm{~cm})$ was divided into an interaction zone and two opposing corner zones. A mesh plastic target box $(10 \times 4.5 \mathrm{~cm})$ was placed in the interaction zone. A test mouse 
was allowed to roam around the open-field arena for 2.5 min with no social target (CD1 mouse) in the mesh box (denoted as 'no target' in figures showing results of social interaction experiments). After this, a novel CD1 mouse was placed in a metal mesh plastic target box in the interaction zone (denoted as 'target' in figures showing results of social interaction experiments) and the test mouse was placed back into the open arena for another $2.5 \mathrm{~min}$. Using the stopwatch, the amount of time spent in the interaction zone (defined as the $8 \mathrm{~cm}$ wide area surrounding the wire mesh cage) was measured both with and without the social target. ${ }^{32}$

\section{Behavioral tests}

Locomotion: the mice were placed in experimental cages (length $\times$ width $x$ height: $560 \times 560 \times 330 \mathrm{~mm}$ ). ${ }^{33-40}$ Locomotor activity of mice was counted using SCANET MV-40 (MELQUEST, Toyama, Japan), and cumulative exercise was recorded for $60 \mathrm{~min}$. The cages were cleaned between the testing sessions. Tail suspension test: the mice were taken from their home cages and a small piece of adhesive tape was placed approximately $2 \mathrm{~cm}$ from the tip of their tails. A single hole was punched in the tape and the mice were hung individually on a hook. The immobility time of each mouse was recorded for $10 \mathrm{~min}$. They were considered immobile only when they hung passively and completely motionless. Forced swimming test: the mice were placed individually in a cylinder (diameter: $23 \mathrm{~cm}$; height: $31 \mathrm{~cm}$ ) containing $15 \mathrm{~cm}$ of water, maintained at $23 \pm 1^{\circ} \mathrm{C}$. They were tested in an automated forced-swim apparatus using SCANET MV-40. Immobility time was calculated from activity time as total active time using the apparatus analysis software. Cumulative immobility time was scored for 6 min during the test. Sucrose preference test: the mice were habituated to a $1 \%$ sucrose solution for $48 \mathrm{~h}$ before the test day. They were deprived of water and food for $4 \mathrm{~h}$, followed by a preference test spanning $1 \mathrm{~h}$ with water and $1 \%$ sucrose, delivered from identical bottles. The bottles containing water and sucrose were weighed before and at the end of this period and the sucrose preference (\%) was determined.

\section{Enzyme-linked immunosorbent assay}

The blood samples were obtained via a cardiac puncture 1 or 7 days after MR16-1 administration. The blood was centrifuged at $2000 \mathrm{~g}$ for $20 \mathrm{~min}$ to generate serum samples. The serum samples were diluted 10 -fold with ELISA (enzyme-linked immunosorbent assay) diluent solution (eBioscience, San Diego, CA, USA). The serum levels of tumor necrosis factor-a (TNF-a), interleukin-1 $\beta$ (IL-1 $\beta$ ) and interleukin-6 (IL-6) were measured using a Ready-SET-Go ELISA kit (eBioscience) according to the manufacturer's instructions.

\section{Western blot analysis}

The brain samples of CA1, CA3 and DG of the hippocampus, prefrontal cortex (PFC) and nucleus accumbens (NAc) from mice were dissected as previously reported. ${ }^{33,34,37-40}$ The tissue samples were homogenized in Laemmli lysis buffer. The aliquots $(10 \mu \mathrm{g})$ of protein were measured using a DC protein assay kit (Bio-Rad, Hercules, CA, USA) and incubated for 5 min at $95^{\circ} \mathrm{C}$, with an equal volume of $125 \mathrm{~mm}$ Tris/ $\mathrm{HCl}, \mathrm{pH} 6.8,20 \%$ glycerol, $0.1 \%$ bromophenol blue, $10 \% \beta$-mercaptoethanol and $4 \%$ sodium dodecyl sulfate, and subjected to sodium dodecyl sulfate polyacrylamide gel electrophoresis using 10\% mini-gels (Mini-PROTEAN TGX Precast Gel; BioRad). The proteins were transferred onto polyvinylidene difluoride membranes using a Trans Blot Mini Cell (Bio-Rad). For immunodetection, the blots were blocked with $2 \%$ bovine serum albumin in TBST (TBS+0.1\% Tween-20) for $1 \mathrm{~h}$ at room temperature and kept with primary antibodies overnight at $4{ }^{\circ} \mathrm{C}$. The following primary antibody was used: postsynaptic density protein 95 (PSD-95; $1 \mu \mathrm{g} \mathrm{ml}^{-1}$ Invitrogen, Carlsbad, CA, USA). The next day, blots were washed three times in TBST and incubated with horseradish peroxidase conjugated anti-rabbit antibody (1:5000) for $1 \mathrm{~h}$, at room temperature. After the final three washes with TBST, bands were detected using enhanced chemiluminescence plus the Western Blotting Detection system (GE Healthcare Bioscience, Tokyo, Japan). The blots then were incubated in the stripping buffer ( $2 \%$ sodium dodecyl sulfate, $100 \mathrm{~mm}$ $\beta$-mercaptoethanol and $62.5 \mathrm{mmTris}-\mathrm{HCl}, \mathrm{pH} \mathrm{6.8)}$ for $30 \mathrm{~min}$ at $60^{\circ} \mathrm{C}$ and then washed three times with TBST. The stripped blots were kept in blocking solution for $1 \mathrm{~h}$ and incubated with the primary antibody directed against a-amino-3-hydroxy-5-methyl-4-isoxazolepropionic acid receptor (AMPAR) 1 (GluA1; $1 \mu \mathrm{g} \mathrm{ml}^{-1}$ Abcam, Cambridge, MA, USA) and $\beta$-actin. Images were captured with a Fuji LAS3000-mini imaging system (Fujifilm, Tokyo, Japan) and immunoreactive bands were quantified.
Golgi staining

Golgi staining was performed using the FD Rapid GolgiStain Kit (FD Neuro Technologies, Columbia, MD, USA), following the manufacturer's instructions..$^{38-40}$ The animals were deeply anesthetized with isoflurane and pentobarbital, and the brains were removed from the skulls and rinsed in double distilled water. The brains were immersed in the impregnation solution, prepared by mixing equal volumes of solutions $A$ and $B$ overnight, and then stored in a fresh solution for 2 weeks in the dark. The brains were transferred into solution $\mathrm{C}$ overnight and then stored in fresh solution at $4{ }^{\circ} \mathrm{C}$ for 1 week in the dark. Coronal brain sections $(100 \mu \mathrm{m}$ thickness) were cut on a cryostat (3050S, Leica Microsystems, Wetzlar, Germany), with the chamber temperature set at $-20^{\circ} \mathrm{C}$. Each section was mounted in solution $\mathrm{C}$ on saline-coated microscope slides. After absorption of excess solution, the sections were dried naturally at room temperature. The dried sections were processed following the manufacturer's instructions. Briefly, images of dendrites within CA1, CA3 and DG of the hippocampus, PFC and NAc were captured using a $\times 100$ objective with a Keyence BZ-9000 Generation II microscope (Osaka, Japan). The spines were counted along the CA1, CA3, DG, PFC and NAC dendrites starting from their points of origin from the primary dendrite, as previously reported. ${ }^{38-43}$ For spine density measurements, all clearly evaluable areas containing 50-100 $\mu \mathrm{m}$ of secondary dendrites from each imaged neuron were used. To determine relative spine density, the spines on multiple dendritic branches from a single neuron were counted to obtain an average spine number per $10 \mu \mathrm{m}$. For spine number measurements, only spines that emerged perpendicular to the dendritic shaft were counted. Three neurons per section, three sections per animal and six animals were analyzed. The average value for each region in each individual was obtained. These individual averages were then combined to yield a grand average for each region.

16S rRNA analysis of fecal samples

The fecal samples were collected 7 days after MR16-1 (or control lgG) administration and placed in $1.5 \mathrm{ml}$ tubes, snap-frozen on dry ice and stored at $-80^{\circ} \mathrm{C}$. The $16 \mathrm{~S}$ rRNA analysis of fecal samples was performed at Takara Bio (Shiga, Japan). The DNA extraction was performed using the MoBio Powerlyzer Powersoil DNA Isolation Kit (MoBio Laboratories, Carlsbad, CA, USA). The V4 hypervariable region of the bacterial 16S rRNA gene was amplified from the fecal DNA extracts using modified universal bacterial primer pairs 515F (5'-TCGTCGGCAGCGTCAGATGTGTATAAGAG ACAGGTGCCAGCMGCCGCGGTAA-3') and 806R (5'-GTCTCGTGGGCTCGGAG ATGTGTATAAGAGACAGGGACTACHVGGGTWTCTAAT-3') with Illumina adaptor overhang sequences. Amplicons were generated, cleaned, indexed and sequenced according to the Illumina MiSeq 16S Metagenomic Sequencing Library Preparation protocol (http://support.illumina.com/ downloads/16s_metagenomic_sequencing_library_preparation.html) with certain modifications.

Sequencing data were combined and sample identification assigned to multiplexed reads using the MOTHUR software environment. ${ }^{44}$ The data were denoised; low quality sequences, pyrosequencing errors, and chimeras were removed, and then sequences were clustered into operational taxonomic units (OTUs) at $97 \%$ identity using the CD-HITOTU pipeline (available from http://eeizhong-lab.ucsd.edu/cd-hit-otu). ${ }^{45}$ OTUs containing fewer than four reads per individual diet/animal combination were excluded due to the likelihood of there being a sequencing artifact. The samples were normalized by randomly resampling sequences used to the lowest number of sequences per sample (each diet/ animal combination) using Daisychopper (http://www.festinalente.me/ bioinf/). Taxonomic classification of OTUs was conducted using the Ribosomal Database Project Classifier. ${ }^{46}$

\section{Statistical analysis}

The data are shown as mean \pm s.e.m. Analysis was performed using PASW Statistics 20 (formerly SPSS statistics; SPSS, Tokyo, Japan). Comparisons between groups were performed using one-way analysis of variance followed by the post hoc least significant difference test or two-way analysis of variance; when appropriate, post hoc comparisons were performed using the unpaired $t$-test. $P$-values $<0.05$ were considered statistically significant. 

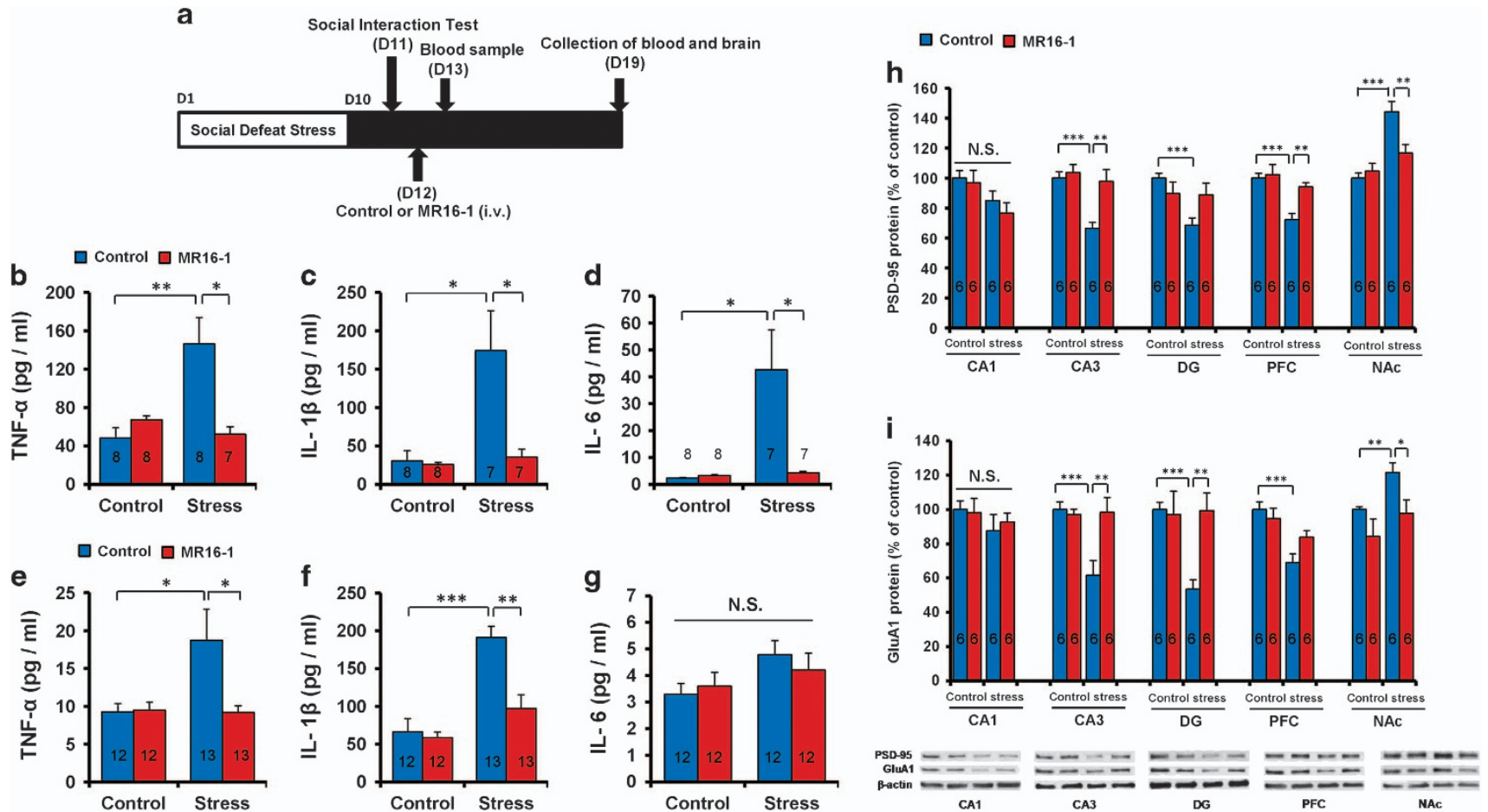

Figure 2. Anti-inflammatory effects of MR16-1 in serum and the expression of synaptic proteins in the brain of susceptible mice after social defeat stress. (a) Schedule of social defeat stress (10 days), social interaction test, drug treatment and sample collection. Samples of blood and brain were collected 1 day and 7 days after intravenous (i.v.) injection of MR16-1. Serum levels of tumor necrosis factor (TNF)- $\alpha$, interleukin (IL)$1 \beta$ and IL- 6 were measured using enzyme-linked immunosorbent assay (ELISA). The levels of PSD-95 and GluA1 in the brain regions were measured using western blot. (b-d) Serum levels of TNF- $\alpha, \mathrm{IL}-1 \beta$ and IL-6 on day 13 (1 day after injection). Two-way analysis of variance (ANOVA) revealed the results (TNF- $\alpha(b)$, Stress: $F_{1,30}=5.541, P=0.026 ;$ MR16-1: $F_{1,30}=4.608, P=0.041 ;$ interaction: $F_{1,30}=10.326, P=0.003$ ), (IL-1 $\beta$ (c), Stress: $F_{1,29}=9.026, P=0.006 ;$ MR16-1: $F_{1,30}=7.935, P=0.009$; interaction: $\left.F_{1,30}=6.938, P=0.014\right)$, (IL-6 (d), Stress: $F_{1,30}=7.868$, $P=0.009$; MR16-1: $\mathrm{F}_{1,30}=6.487, P=0.017$; interaction: $\left.\mathrm{F}_{1,30}=7.179, P=0.013\right)$. Data are shown as the mean \pm s.e.m. $(n=7$ or 8$) .{ }^{*} P<0.05$ and ${ }^{* *} P<0.01$ compared with the control-treated stress group. (e-g) Serum levels of TNF- $\alpha$, IL-1 $\beta$ and IL-6 on day 19 (7 days after injection). Twoway ANOVA revealed the results (TNF- $\alpha(\mathbf{e})$, Stress: $F_{1,47}=4.192, P=0.047 ;$ MR16-1: $F_{1,47}=4.301, P=0.044$; interaction: $F_{1,47}=4.744, P=0.035$ ), (IL-1 $\beta$ (f), Stress: $F_{1,47}=29.068, P<0.001$; MR16-1: $F_{1,47}=11.296, P=0.002$; interaction: $\left.F_{1,47}=8.032, P=0.007\right)$, (IL-6 (g), Stress: $F_{1,45}=4.532$, $P=0.039$; MR16-1: $F_{1.45}=0.006, P=0.939$; interaction: $\left.F_{1.45}=0.405, P=0.528\right)$. Data are shown as the mean \pm s.e.m. $(n=12$ or 13$) . * P<0.05$, ${ }^{* *} P<0.01,{ }^{* * *} P<0.001$ compared with the control-treated stressed group. (h and i) Western blot of PSD-95 and GluA1 in the CA1, CA3 and DG of hippocampus, prefrontal cortex (PFC) and nucleus accumbens (NAC). (h) Two-way ANOVA revealed the results $\left(C A 1\right.$, Stress: $F_{1,22}=3.256$, $P=0.088 ;$ MR16-1: $F_{1,22}=1.414, P=0.250$; interaction: $\left.F_{1,22}=0.536, P=0.474\right)$, (CA3, Stress: $F_{1,22}=11.880, P=0.003 ; M R 16-1: F_{1,22}=9.458$, $P=0.006$; interaction: $F_{1,22}=5.788, P=0.026$ ), (DG, Stress: $F_{1,21}=6.180, P=0.023 ;$ MR16-1: $F_{1,21}=0.599, P=0.449$; interaction: $F_{1,21}=5.523$, $P=0.030)$, (PFC, Stress: $\mathrm{F}_{1,22}=15.933, P=0.001$, MR16-1: $\mathrm{F}_{1,22}=7.278, P=0.014$; interaction: $\left.\mathrm{F}_{1,22}=4.772, P=0.042\right)$, (NAC, Stress: $\mathrm{F}_{1,22}=26.386$, $P=0.001$; MR16-1: $F_{1,22}=4.429, P=0.049$; interaction: $F_{1,22}=8.705, P=0.008$ ). (i) Two-way ANOVA revealed the results (CA1, Stress: $F_{1,22}=1.442, P=0.245 ;$ MR16-1: $F_{1,22}=0.043, P=0.839$; interaction: $\left.F_{1,22}=0.220, P=0.645\right)$, $\left(C A 3\right.$, Stress: $F_{1,22}=7.600, P=0.013 ; M R 16-1$ : $\mathrm{F}_{1,22}=6.257, P=0.022$; interaction: $\left.\mathrm{F}_{1,22}=8.696, P=0.008\right)$, (DG, Stress: $\mathrm{F}_{1,22}=5.075, P=0.037$; MR16-1: $\mathrm{F}_{1,22}=4.731, P=0.043$; interaction: $\left.\mathrm{F}_{1,22}=6.105, P=0.024\right),\left(P F C\right.$, Stress: $F_{1,22}=18.398, P<0.001 ; M R 16-1: F_{1,22}=0.979, P=0.335$; interaction: $\left.F_{1,22}=4.223, P=0.054\right),(N A c$, Stress: $F_{1,22}=5.590, P=0.029 ;$ MR16-1: $F_{1,22}=7.094, P=0.015$; interaction: $\left.F_{1,22}=0.293, P=0.595\right)$. The values are expressed as percentages relative to those in the control mice. The values represent the mean \pm s.e.m. $(n=5$ or 6$)$. ${ }^{*} P<0.05,{ }^{* *} P<0.01,{ }^{* * *} P<0.001$ compared with the controltreated stress group. The number in the column is the number of mice. NS, not significant.

\section{RESULTS}

Antidepressant effects of MR16-1 in the social defeat stress model To examine the antidepressant effects of MR16-1 in the social defeat stress model, intravenous injection of MR16-1 or control was administered $60 \mathrm{~min}$ before the locomotion test (Figure 1a). No effect was observed in spontaneous locomotion in the four groups (Figure 1b). In the tail suspension test, MR16-1 significantly attenuated the increased immobility time observed in susceptible mice after social defeat stress (Figure 1c). In the forced swimming test, there was no significant interaction (Figure 1d). In the 1\% sucrose preference test, MR16-1 significantly attenuated decreased sucrose preference in susceptible mice 3 and 6 days after a single dose (Figures $1 \mathrm{e}$ and $\mathrm{f}$ ). The results suggest that intravenous injection of MR16-1 showed rapid and sustained antidepressant effects in a social defeat stress model.

It was reported that peripheral, but not brain, IL-6 might be involved in the depression-like phenotypes in rodents. ${ }^{8,13,18}$ Therefore, we examined whether intracerebroventricular injection of MR16-1 showed antidepressant effects in the social defeat stress model (Figure 1g). No effect was observed in spontaneous locomotion in the four groups (Figure 1h). In the tail suspension test and forced swimming test, intracerebroventricular injection of MR16-1 did not attenuate the increased immobility time of susceptible mice (Figures $1 \mathrm{i}$ and j). Moreover, MR16-1 did not affect the decreased sucrose preference of susceptible mice (Figure $1 \mathrm{k}$ ). These results suggest that intracerebroventricular injection of MR16-1 did not show antidepressant effects in the social defeat stress model. Collectively, it is likely that the blockade of the IL- 6 receptor in the periphery may be involved in the antidepressant effects of MR16-1.

Anti-inflammatory effects of MR16-1 in the serum of susceptible mice after social defeat stress

To examine the effects of MR16-1 on serum levels of proinflammatory cytokines in susceptible mice after social defeat 


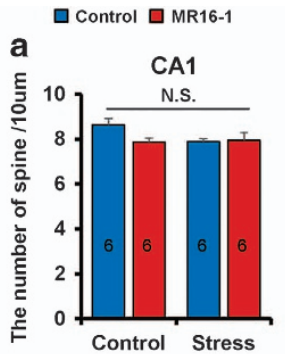

b
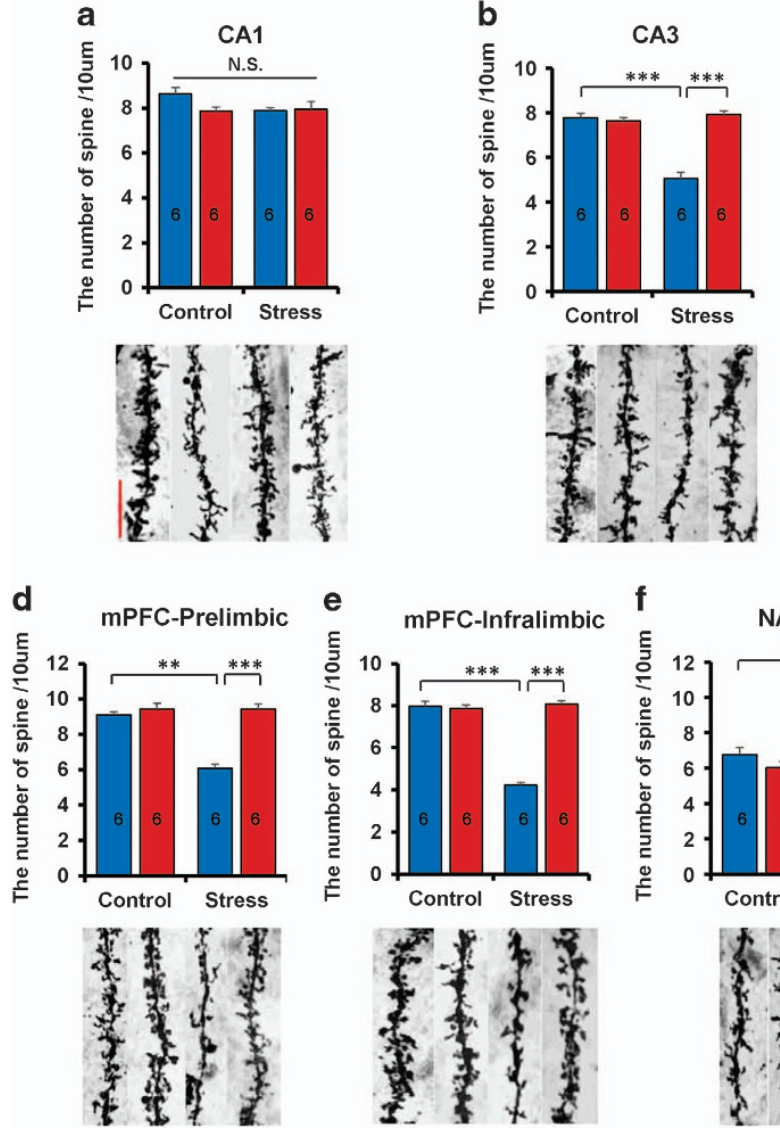

mPFC-Infralimbic
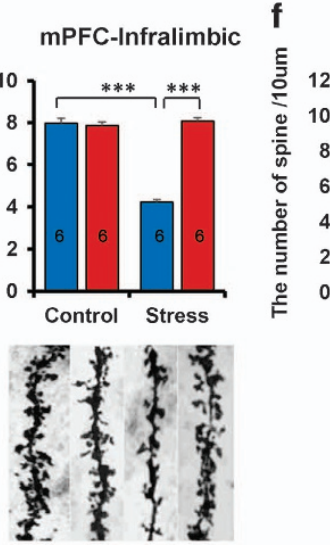
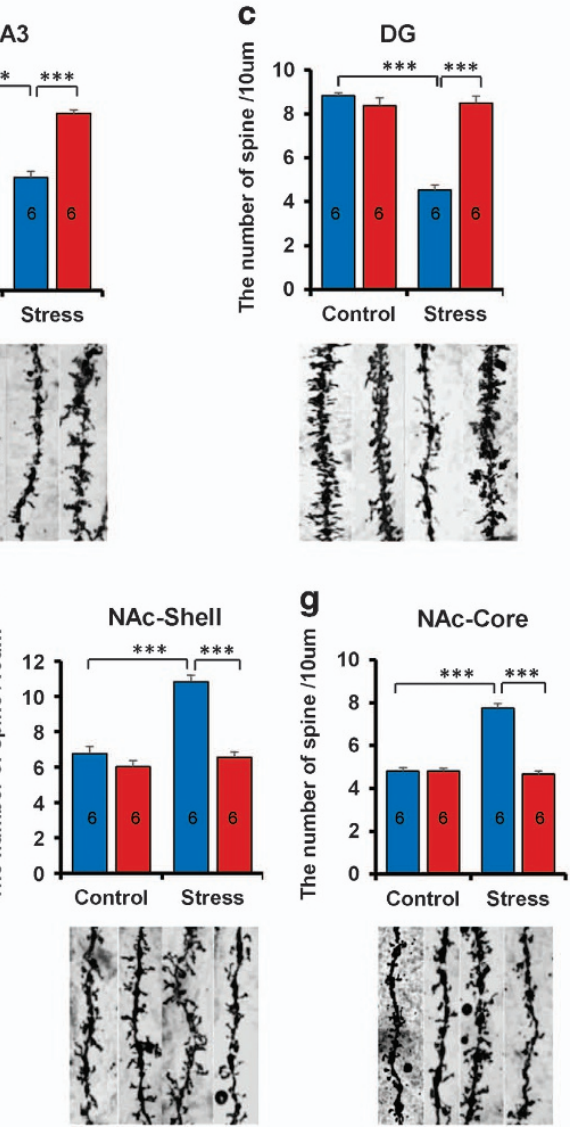

Figure 3. Effects of MR16-1 on alterations in the spine density of brain regions of susceptible mice after social defeat stress. (a-g) Representative photomicrographs of Golgi-Cox stained pyramidal neurons in the CA1, CA3 and DG of the hippocampus, prelimbic and infralimbic of medial prefrontal cortex (PFC), nucleus accumbens (NAc) shell and NAc core from animals of each group. Scale bar, $10 \mu \mathrm{m}$. Twoway analysis of variance (ANOVA) revealed the following results: (CA1 (a), Stress: $F_{1,23}=2.949, P=0.101 ; M R 16-1: F_{1,23}=2.215, P=0.152$; interaction: $\left.F_{1,23}=1.887, P=0.185\right)$, (CA3 (b), Stress: $F_{1,23}=34.883, P<0.001$; MR16-1: $F_{1,23}=43.261, P<0.001$; interaction: $F_{1,23}=52.541$, $P<0.001)$, (DG (c), Stress: $F_{1,23}=60.089, P<0.001 ;$ MR16-1: $F_{1,23}=42.512, P<0.001$; interaction: $\left.F_{1,23}=66.634, P<0.001\right)$, (mPFC PrL (d), Stress: $\mathrm{F}_{1,23}=28.073, P<0.001$; MR16-1: $\mathrm{F}_{1,23}=41.098, P<0.001$; interaction: $\left.\mathrm{F}_{1,23}=28.073, P<0.001\right),\left(\mathrm{mPFC} \mathrm{IL}(\mathbf{e})\right.$, Stress: $\mathrm{F}_{1,23}=98.468, P<0.001$; MR16-1: $F_{1,23}=111.264, P<0.001$; interaction: $F_{1,23}=124.841, P<0.001$ ), (NAc shell (f), Stress: $F_{1,23}=29.435, P<0.001 ; M R 16-1: F_{1,23}=36.049$, $P<0.001$; interaction: $F_{1,23}=16.416, P<0.001$ ), (NAc core $(g)$, Stress: $F_{1,23}=72.553, P<0.001$; MR16-1: $F_{1,23}=87.632, P<0.001$; interaction: $\left.\mathrm{F}_{1,23}=87.632, P<0.001\right)$. The values represent the mean \pm s.e.m. $(n=6)$. ${ }^{* *} P<0.01,{ }^{* * *} P<0.001$ compared with the control-treated stress group. The number in the column is the number of mice. NS, not significant.

stress, blood samples were collected 1 and 7 days after intravenous injection (Figure 2a). Serum levels of TNF- $a$, IL-1 $\beta$ and IL-6 in susceptible mice were significantly higher than those in the control group 1 day after injection (Figures 2b-d). MR16-1 significantly attenuated the increased levels of TNF- $a, I L-1 \beta$ and IL-6 in susceptible mice 1 day after injection (Figures 2b-d). Furthermore, MR16-1 significantly attenuated the increased levels of TNF- $\alpha$ and IL-1 $\beta$ in susceptible mice 7 days after injection (Figures $2 e$ and f). However, there were no changes of serum IL-6 levels among the four groups 7 days after injection (Figure $2 \mathrm{~g}$ ).

Levels of PSD-95 and GluA1 in the brain regions after a single injection of MR16-1

We performed western blot of the synaptogenesis markers, PSD-95 and GluA1 in the brain regions, which were collected 7 days after intravenous injection of MR16-1 or control. Susceptible mice showed decreased protein levels of PSD-95 in the hippocampus (CA3 and DG) and PFC, whereas they showed increased levels of PSD-95 in the NAc (Figure 2h). MR16-1 significantly attenuated alterations in the levels of PSD-95 in the CA3, PFC and NAc (Figure 2h). Furthermore, susceptible mice showed decreased protein levels of GluA1 in the hippocampus (CA3 and DG), whereas they showed increased levels of GluA1 in the NAC (Figure 2i). MR16-1 significantly attenuated alterations in the levels of GluA1 in the CA3, DG and NAc (Figure 2i). Collectively, MR16-1 could normalize alterations in the synaptic proteins (PSD-95 and GluA1) in the brain regions of susceptible mice.

Effect of MR16-1 on alterations in the dendritic spine density in the brain regions of susceptible mice after social defeat stress Alterations in the dendritic length and spine density in the hippocampus, PFC and NAc have an important role in the pathogenesis of depression, and antidepressant treatment can block or reverse these changes. ${ }^{41-43,47,48,49}$ Susceptible mice showed a decreased spine density in the hippocampus (CA3 and DG) and medial PFC (prelimbic (PrL) and infralimbic (IL) regions), whereas these mice had an increased spine density in the NAc shell and core (Figures 3a-g). Treatment with MR16-1 significantly attenuated the decreased spine density in the CA3, DG and MPFC (PrL and IL) of susceptible mice (Figures 3a-e). In contrast, treatment with MR16-1 significantly attenuated increased spine density in the NAc shell and core of susceptible 


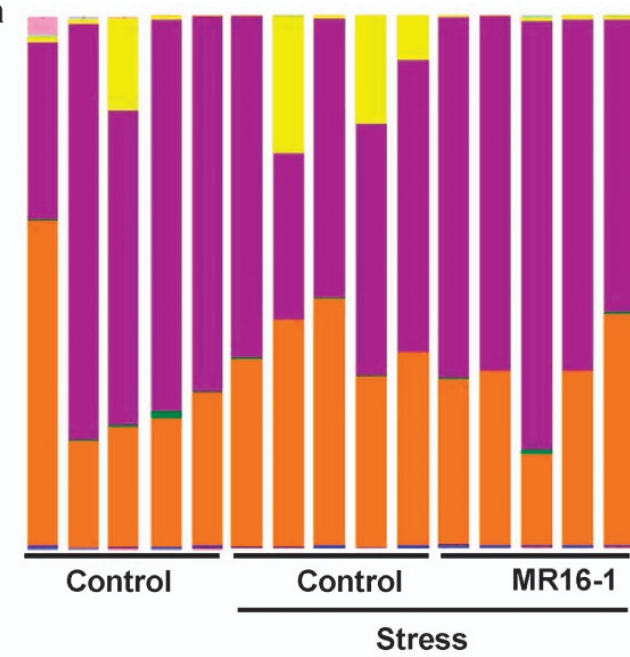

b

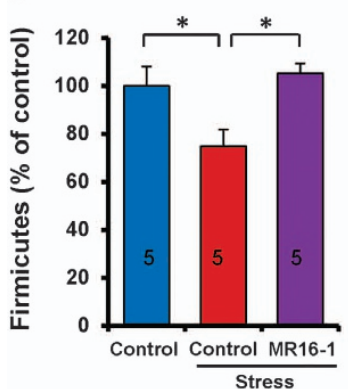

c

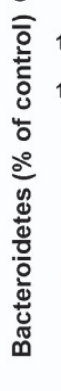

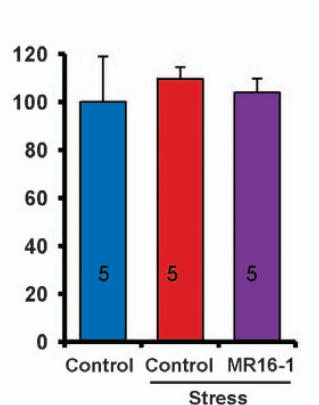

d

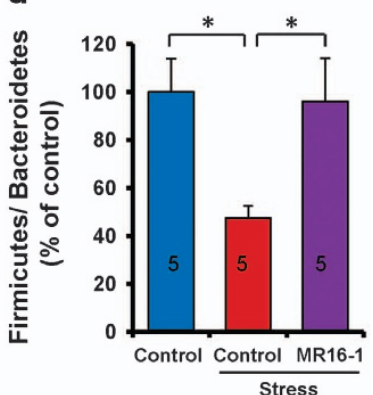

Figure 4. Effects of MR16-1 on alterations in the composition of gut microbiome in phylum of susceptible mice after social defeat stress. (a) Phylum. (b) Levels of Firmicutes. (c) Levels of Bacteroidetes. (d) Ratio of Firmicutes to Bacteroidetes. One-way analysis of variance (ANOVA) revealed the results (Firmicutes (b), $\mathrm{F}_{1,14}=5.227, P=0.023$ ), (Bacteroidetes $(\mathbf{c}), \mathrm{F}_{1,14}=0.137, P=0.873$ ), (Firmicutes/Bacteroidetes ratio (d); $\left.\mathrm{F}_{1,14}=3.964, P=0.048\right)$. Data are shown as the mean \pm s.e.m. $(n=5) .{ }^{*} P<0.05$ compared with the control-treated stress group. The number in the column is the number of mice. NS, not significant.

mice (Figures $3 f$ and $g$ ). These results suggest that MR16-1 could induce antidepressant-like effects by normalizing alterations in the spine density in these brain regions of susceptible mice after social defeat stress.

Antidepressant effects of MR16-1 by normalizing the altered composition of the gut microbiome

For the gut microbiome analysis using $16 \mathrm{~S}$ ribosomal RNA gene sequencing, fecal samples were collected 7 days after intravenous injection of MR16-1 or control. Susceptible mice showed decreased levels of Firmicutes and decreased Firmicutes/Bacteroidetes ratio at the phylum level (Figures 4a-d). MR16-1 significantly attenuated a decreased level of Firmicutes and decreased Firmicutes/Bacteroidetes ratio in susceptible mice after social defeat stress (Figures $4 a-d$ ).

At the genus level, susceptible mice showed increased levels of Staphylococcus and decreased levels of Butyricicoccus and Oscillospira (Figures 5a, b, d and e). Furthermore, susceptible mice revealed a nonsignificant trend toward an increased level of Sutterella (Figures $5 \mathrm{a}$ and c). MR16-1 significantly improved the increased levels of Sutterella and decreased levels of Oscillospira in susceptible mice (Figures $5 \mathrm{c}$ and e). In addition, MR16-1 nonsignificantly tended to alter the levels of Staphylococcus and Butyricicoccus (Figures $5 b$ and d).

\section{DISCUSSION}

The major findings of this study are that peripheral, but not brain, IL- 6 has an important role in the depression-like phenotype after social defeat stress and that gut microbiota may have a role in the antidepressant effects of anti-IL-6 receptor MR16-1. First, intravenous injection of MR16-1 showed rapid and long-lasting antidepressant effects in the social defeat stress model, although intracerebroventricular injection of MR16-1 did not show antidepressant-like effects in the same model. Second, MR16-1 could attenuate alterations in the synaptic proteins (PSD-95 and GluA1) and dendritic spine density in the brain regions of susceptible mice after social defeat stress. Third, MR16-1 could normalize alterations in the gut microbiota composition in susceptible mice after social defeat stress. These findings suggest that increases in the pro-inflammatory cytokines and altered composition of gut microbiota induced by social defeat stress might have an important role in the pathogenesis of depression and that the gut-microbiota-brain axis may be implicated in the rapid-onset and long-lasting antidepressant actions of anti-IL-6 receptor antibody. The blockade of IL-6 receptor by the humanized anti-IL- 6 receptor antibody tocilizumab has been used in the treatment of rheumatoid arthritis, ${ }^{50}$ and a human anti-IL- 6 monoclonal antibody, sirukumab, has been under evaluation in patients, including those with rheumatoid arthritis. Therefore, it is likely that a humanized anti-IL-6 receptor antibody (for example, tocilizumab) or a human anti-IL-6 monoclonal antibody (for example, sirukumab) would be potential therapeutic drugs for the blockade of IL- 6 signaling in depressed patients with higher blood levels of IL-6.

Social defeat stress has been reported to increase IL- 6 release in the serum of patients who subsequently developed a depressionlike phenotype. ${ }^{8}$ In addition, serum levels of IL-6 strongly 

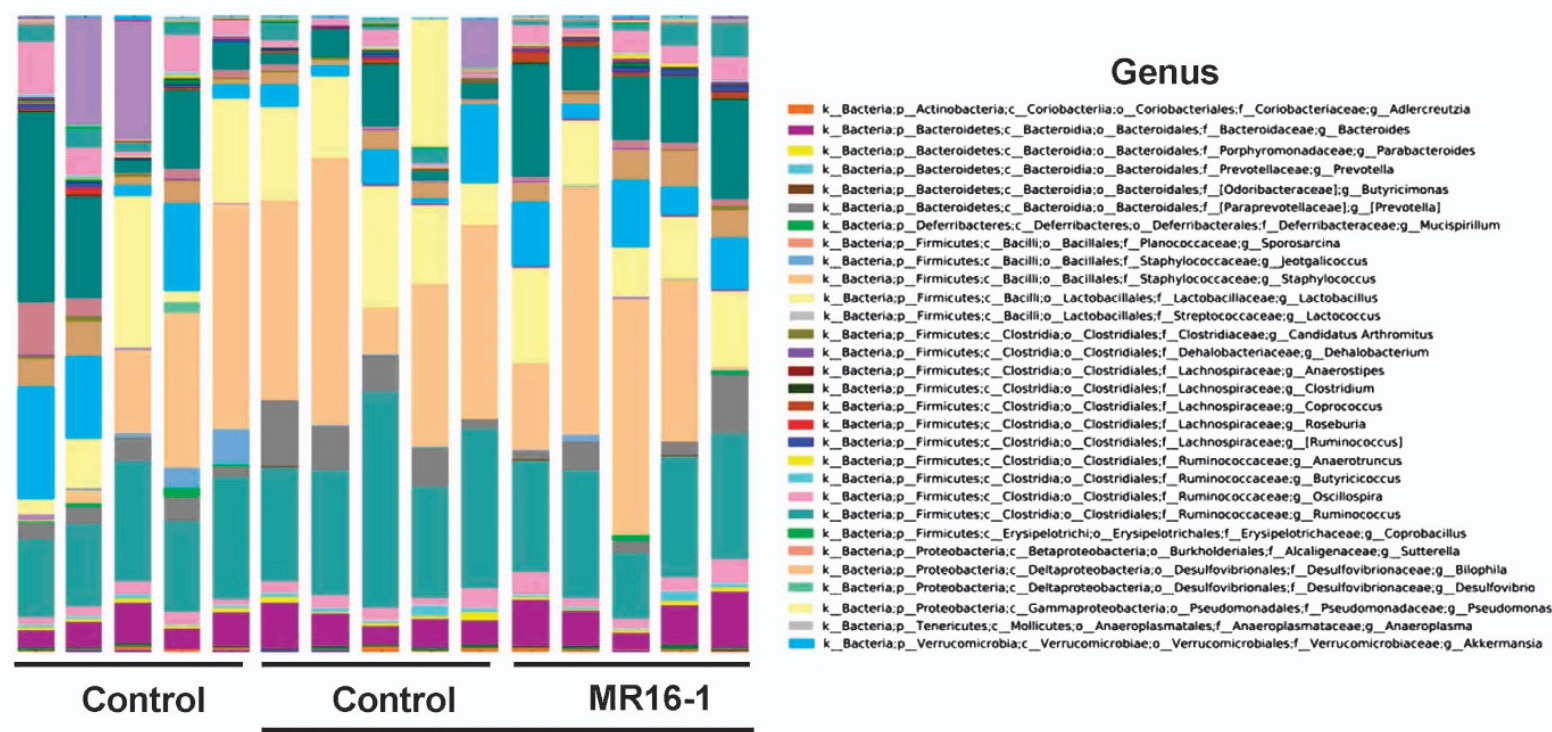

\section{Stress}
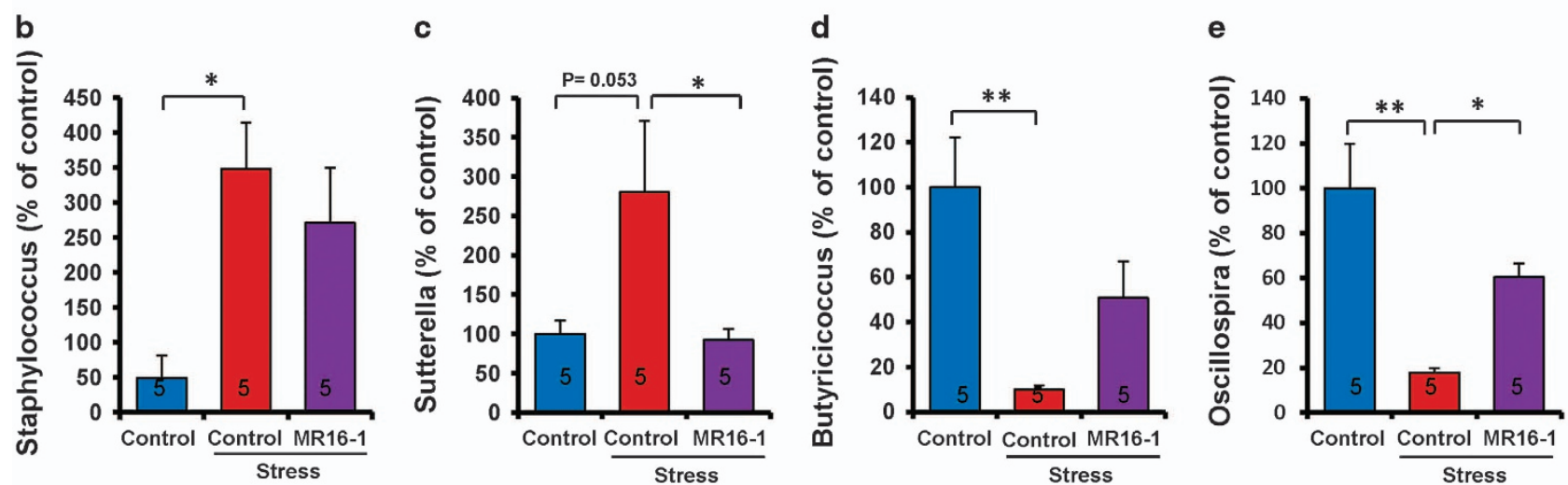

Figure 5. Effects of MR16-1 on alterations in the composition of gut microbiome in genus of susceptible mice after social defeat stress. (a) Genus. (b) Levels of Staphylococcus. (c) Levels of Sutterella. (d) Levels of Butyricicoccus. (e) Levels of Oscillospira. One-way analysis of variance (ANOVA) revealed the results (Staphylococcus $\left.(\mathbf{b}), \mathrm{F}_{1,14}=4.104, P=0.044\right)$, (Sutterella $\left.(\mathbf{c}), \mathrm{F}_{1,14}=4.505, P=0.037\right)$, (Butyricicoccus $(\mathbf{d}), \mathrm{F}_{1,14}=6.727$, $P=0.011)$, (Oscillospira $\left.(\mathbf{e}), \mathrm{F}_{1,14}=9.762, P=0.003\right)$. Data are shown as the mean \pm s.e.m. $(n=5)$. ${ }^{*} P<0.05$ and ${ }^{* *} P<0.01$ compared with the control-treated stress group. The number in the column is the number of mice. NS, not significant.

correlated with social interaction behavior following repeated social defeat stress. Stress-susceptible bone marrow chimeras revealed increased social avoidance behavior after exposure to either sub-threshold repeated social defeat stress, or a purely emotional stressor, termed witness defeat. ${ }^{8}$ We also reported that alterations in the peripheral, but not brain, IL-6 might contribute to resilience versus susceptibility to inescapable stress in the rat learned helplessness model. ${ }^{18}$ These results suggest that peripheral, but not brain, IL- 6 might be involved in the depression-like phenotype in rodents. ${ }^{13,14,18}$ Thus, it is unlikely that the absence of beneficial effects with intracerebroventricular administration may be related to a lack of drug penetration into the parenchymal brain tissues implicated in depression. Collectively, it is likely that the IL-6 receptor antibody MR16-1 showed rapid and long-lasting antidepressant effects in the social defeat stress model by inhibiting IL-6 receptor in the periphery. In addition, IL-6 knockout mice showed resilience to stress-induced development of depression-like behaviors, ${ }^{51}$ suggesting the role of IL-6 in depression.

Accumulating evidence suggests that abnormalities in the gut microbiota composition have a causative role in the pathogenesis of depression. ${ }^{20,21,25,26,27,52}$ At the phylum level, Firmicutes and Bacteroidetes constitute the largest portion of the mouse and human gut microbiome. The Firmicutes/Bacteroidetes ratio is shown to be of significant relevance in signaling human gut microbiota status. ${ }^{53,54}$ For example, obesity is associated with changes in the relative abundance of these two dominant bacterial divisions. ${ }^{53,54}$ Chronic restraint stress in mice caused a nonsignificant trend toward increased Firmicutes/Bacteroidetes ratio, and the anti-inflammatory drug minocycline attenuated increased Firmicutes/Bacteroidetes ratio. ${ }^{26}$ Furthermore, increased Firmicutes/Bacteroidetes ratio was reported in patients with irritable bowel syndrome. ${ }^{55}$ Thus, increased Firmicutes/Bacteroidetes ratio, caused by the increase of Firmicutes or decrease of Bacteroidetes, has been widely considered a signature of gut dysbiosis. Conversely, a decreased Firmicutes/Bacteroidetes ratio was reported to be related to weight loss. ${ }^{54}$ Moreover, the Firmicutes/Bacteroidetes ratio is also linked to overall changes in bacterial profiles at different stages of life. ${ }^{56} \mathrm{~A}$ recent study showed that syringaresinol (a polyphenolic lignan) significantly improved decreased Firmicutes/Bacteroidetes ratio in middle-aged mice, suggesting that syringaresinol may rejuvenate the immune 
system through modulation of gut integrity and microbiota diversity as well as composition in middle-aged mice. ${ }^{57}$ In this study, we found decreased number of Firmicutes and decreased Firmicutes/Bacteroidetes ratio in susceptible mice after social defeat stress, suggesting that a decreased Firmicutes/Bacteroidetes ratio might have a role in the depression-like phenotype. Interestingly, MR16-1 could attenuate the decreased number of Firmicutes and decreased Firmicutes/Bacteroidetes ratio in susceptible mice. Inflammatory cytokines such as IL- 6 and IL-1 $\beta$ in the gut microbiota have a role in inflammation diseases. ${ }^{58}$ Collectively, it is likely that MR16-1 may show antidepressant-like effects by normalizing abnormalities in Firmicutes (or Firmicutes/Bacteroidetes ratio) through the modulation of the immune system. Nonetheless, further detailed studies underlying the role of gut microbiota in the antidepressant actions of MR16-1 are needed.

At the genus level, Staphylococcus and Sutterella are genera of gram-positive and gram-negative bacteria, respectively. An increased number of Sutterella was reported in the feces of children with autism spectrum disorder, suggesting an imbalance in the gut microbiota in children with autism spectrum disorder. ${ }^{59}$ We found increased numbers of Staphylococcus and Sutterella in susceptible mice after social defeat stress. Although it is not yet evident what the consequences of increased number of fecal Staphylococcus (or Sutterella) indicates, it is possible that, under stress conditions, these bacteria may have a role in the depression-like phenotype through infection-induced inflammation. Interestingly, we found that MR16-1 could attenuate the increased numbers of Staphylococcus and Sutterella in susceptible mice after social defeat stress.

Butylate has a key role in maintaining gut health by preventing cell proliferation, suppressing inflammation and providing energy to enterocytes. ${ }^{60}$ Butyricicoccus is a butylate-producing clostridial cluster IV genus whose numbers are reduced in the stool of ulcerative colitis patients. ${ }^{61,62}$ Oscillospira, the clostridial cluster IV of the Firmicutes phylum, also produce butylate. Oscillospira is negatively associated with obesity and inflammatory bowel diseases. $^{63,64}$ In this study, we found that the numbers of Butyricicoccus and Oscillospira decreased in susceptible mice after social defeat stress and that MR16-1 improved decreased numbers of Butyricicoccus and Oscillospira in these mice. Interestingly, decreased numbers of fecal Oscillospira were detected in patients with depression, ${ }^{25}$ suggesting that alterations in Oscillospira number may be involved in the pathogenesis of depression. In addition, sodium butylate, a histone deacetylase inhibitor, showed antidepressant effects in animal models of depression, ${ }^{65}$ suggesting that butylate can be used for treating depression. Considering the role of Butyricicoccus and Oscillospira in the production of butylate, it is likely that decreased numbers of these bacteria may have a role in the pathogenesis of depression. Reconditioning of the gut microbiota through direct supplementation with beneficial bacteria or by indirect stimulation of colonization and proliferation of beneficial bacteria could have a protective role in inflammation-induced depression. However, detailed studies underlying the role of Butyricicoccus and Oscillospira in the pathogenesis of depression are needed.

Growing evidence has suggested that alterations in the synaptic proteins (for instance, PSD-95 and GluA1) and dendritic spine density after social defeat stress have a role in the depression-like phenotype and that the recovery of synaptogenesis by antidepressants has a key role in their antidepressant actions. ${ }^{41-43,47,48,49}$ Intravenous injection of MR16-1 showed a robust antidepressant effect by normalizing decreased levels of PSD-95 and GluA1 in the hippocampus and PFC and increased levels of PSD-95 and GluA1 in the NAc. The rapid-onset and longlasting antidepressant effects of MR16-1 are similar to those of ketamine (or $R$-ketamine) in the social defeat stress model. ${ }^{33-35,42}$

A recent meta-analysis demonstrated higher cerebrospinal fluid levels of IL-6 in depressed patients compared with those in the control subjects. ${ }^{66}$ In this study, we did not find antidepressant effects of MR16-1 after intracerebroventricular injection. Although we did not measure cerebrospinal fluid (or brain) levels of IL- 6 in susceptible mice after intracerebroventricular infusion of MR16-1, it is unlikely that brain IL-6 may have a key role in depression-like phenotypes in rodents. Nonetheless, further studies on the role of IL-6 in the brain and periphery will be needed.

Accumulating evidence suggests that immunomodulation by microbiota is an important pathway that orchestrates the gutmicrobiota-brain axis. ${ }^{21}$ Alterations in immune homeostasis due to host-microbiota interactions can lead to changes in brain function through the hypothalamus-pituitary-adrenal axis, a neuroendocrine, stress-sensing system that could be activated by pro-inflammatory cytokines. ${ }^{21}$ In this study, we found that the blockade of the IL- 6 receptor in the periphery may exert robust antidepressant effects in rodents through immunomodulation by the gut microbiota. Although the precise mechanisms by which the gut microbiota influence brain function remain unclear, there appears to be gut-microbiota-brain axis. Considering the role of the gut microbiota in immunomodulation, it is likely that gutmicrobiota-brain communication may have a role in robust antidepressant actions of anti-IL-6 receptor therapy. Future studies using immunodeficient mice will be necessary to confirm the role of the immune system in the gut-microbiota-brain axis in depression.

In conclusion, these findings suggest that increased peripheral IL-6 signaling and altered composition of the gut microbiota may have an important role in the pathogenesis of depression and that IL-6 receptor antibody MR16-1 showed rapid-onset and longlasting antidepressant effects through the modulation of the immune system. Therefore, it is likely that anti-IL-6 receptor therapy would be a novel therapeutic approach for depressed patients with higher blood levels of IL-6.

\section{CONFLICT OF INTEREST}

The authors declare no conflict of interest.

\section{ACKNOWLEDGMENTS}

This study was partly supported by a Grant-in-Aid for Scientific Research on Innovative Areas of the Ministry of Education, Culture, Sports, Science and Technology, Japan (to KH), and the Strategic Research Program for Brain Sciences from Japan Agency for Medical Research and Development, AMED (to KH). WY was supported by Ishidsu Shun Memorial Scholarship (Tokyo, Japan). QR and CY were supported by Research Fellowship for Young Scientists of the Japan Society for the Promotion of Science. CD was supported by Uehara Memorial Foundation (Tokyo, Japan). MM was supported by Leading Graduate School at Chiba University.

\section{REFERENCES}

1 World Health Organization (WHO). Depression. Fact sheet No. 369/October 2012. Available at http://www.who.int/mediacentre/factsheets/fs369/en/index.html.

2 Dantzer R, O'Connor JC, Freund GG, Johnson RW, Kelly KW. From inflammation to sickness and depression: when the immune system subjugates the brain. Nature Rev Neurosci 2008; 9: 46-57.

3 Miller AH, Maletic V, Raison CL. Inflammation and its discontents: the role of cytokines in the pathophysiology of major depression. Biol Psychiatry 2009; 65: 732-741.

4 Raison CL, Lowry CA, Rook GA. Inflammation, sanitation, and consternation: loss of contact with coevolved, tolerogenic microorganisms and the pathophysiology and treatment of major depression. Arch Gen Psychiatry 2010; 67: 1211-1224.

5 Hashimoto K. Inflammatory biomarkers as differential predictors of antidepressant response. Int J Mol Sci 2015; 16: 7796-7801.

6 Wohleb ES, Franklin T, Iwata M, Duman RS. Integrating neuroimmune systems in the neurobiology of depression. Nat Rev Neurosci 2016; 17: 497-511.

7 Zhang JC, Yao W, Hashimoto K. Brain-derived neurotrophic factor (BDNF)-TrkB signaling in inflammation-related depression and potential therapeutic targets. Curr Neuropharmacol 2016; 14: 721-731. 
8 Hodes GE, Pfau ML, Leboeuf M, Golden SA, Christoffel DJ, Bregman D et al. Individual differences in the peripheral immune system promote resilience versus susceptibility to social stress. Proc Natl Acad Sci USA 2014; 111: 16136-16141.

9 Maes M, Anderson G, Kubera M, Berk M. Targeting classical IL-6 signalling or IL-6 trans-signalling in depression? Expert Opin Ther Targets 2014; 18: 495-512.

10 Young JJ, Bruno D, Pomara N. A review of the relationship between proinflammatory cytokines and major depressive disorder. J Affect Disord 2014; 169: $15-20$.

11 Black C, Miller BJ. Meta-analysis of cytokines and chemokines in suicidality: distinguishing suicidal versus nonsuicidal patients. Biol Psychiatry 2015; 78: 28-37.

12 Fonseka TM, Mclntyre RS, Soczynska JK, Kennedy SH. Novel investigational drugs targeting IL-6 signaling for the treatment of depression. Expert Opin Investig Drugs 2015; 24: 459-475.

13 Yang C, Hashimoto K. Peripheral IL-6 signaling: a promising therapeutic target for depression? Expert Opin Investig Drugs 2015; 24: 989-990.

14 Hodes GE, Ménard C, Russo SJ. Integrating interleukin-6 into depression diagnosis and treatment. Neurobiol Stress 2016; 4: 15-22.

15 Dowlati Y, Herrmann N, Swardfager W, Liu H, Sham L, Reim EK et al A meta-analysis of cytokines in major depression. Biol Psychiatry 2010; 67: 446-457.

16 Strawbridge R, Arnone D, Danese A, Papadopoulos A, Herane Vives A, Cleare AJ. Inflammation and clinical response to treatment in depression: a meta-analysis. Eur Neuropsychopharmacol 2015; 25: 1532-1543.

17 Goldsmith DR, Rapaport MH, Miller BJ. A meta-analysis of blood cytokine network alterations in psychiatric patients: comparisons between schizophrenia, bipolar disorder and depression. Mol Psychiatry 2016; 21: 1696-1709.

18 Yang C, Shirayama Y, Zhang JC, Ren Q, Hashimoto K. Peripheral interleukin- 6 promotes resilience versus susceptibility to inescapable electric stress. Acta Neuropsychiatr 2015; 27: 312-316.

19 Sommer F, Backhed F. The gut microbiota--masters of host development and physiology. Nat Rev Microbiol 2013; 11: 227-238.

20 Kelly JR, Clarke G, Cryan JF, Dinan TG. Brain-gut-microbiota axis: challenges for translation in psychiatry. Ann Epidemiol 2016; 26: 366-372.

21 Fung T, Olson CA, Hsiao E. Interactions between the microbiota, immune and nervous systems in health and disease. Nat Rev Neurosci 2017; 20: 145-155.

22 Tillisch K, Labus J, Kilpatrick L, Jiang Z, Stains J, Ebrat B et al. Consumption of fermented milk product with probiotic modulates brain activity. Gastroenterology 2013; 144: 1394-1401.

23 Dinan TG, Cryan JF. Mood by microbe: towards clinical translation. Genome Med 2016; 6: 36.

24 Foster JA. Gut microbiome and behavior: focus on neuroimmune interactions. Int Rev Neurosci 2016; 131: 49-65.

25 Jiang $\mathrm{H}$, Ling Z, Zhang $\mathrm{Y}$, Mao $\mathrm{H}, \mathrm{Ma} Z$, Yin $\mathrm{Y}$ et al. Altered fecal microbiota composition in patients with major depressive disorder. Brain Behav Immun 2015; 48: $186-194$.

26 Wong $\mathrm{ML}$, Inserra A, Lewis MD, Mastronardi CA, Leong L, Choo J et al. Inflammasome signaling affects anxiety- and depressive-like behavior and gut microbiome composition. Mol Psychiatry 2016; 21: 797-805.

27 Zheng P, Zeng B, Zhou C, Liu M, Fang Z, Xu X et al. Gut microbiome remodeling induces depressive-like behaviors through a pathway mediated by the host's metabolism. Mol Psychiatry 2016; 21: 786-796.

28 Okazaki M, Yamada Y, Nishimoto N, Yoshizaki K, Mihara M. Characterization of anti-mouse interleukin-6 receptor antibody. Immunol Lett 2002; 84: 231-240.

29 Paxinos G, Watson C. The mouse brain in Stereotaxic Coordinates, 4th edition. Academic Press: San Diego, CA, USA, 1998.

30 Golden SA, Covington HE III, Berton O, Russo SJ. A standard protocol for repeated social defeat stress in mice. Nat Protoc 2011; 6: 1183-1191.

31 Zhao T, Huang GB, Muna SS, Bagalkot TR, Jin HM, Chae HJ et al. Effects of chronic social defeat stress on behavior and choline acetyltransferase, 78-kDa glucoseregulated protein, and CCAAT/enhancer-binding protein (C/EBP) homologous protein in adult mice. Psychopharmacology 2013; 228: 217-230.

32 Walsh JJ, Friedman AK, Sun H, Heller EA, Ku SM, Juarez B et al. Stress and CRF gate neural activation of BDNF in the mesolimbic reward pathway. Nature Neurosci 2014; 17: 27-29.

33 Zhang JC, Yao W, Dong C, Yang C, Ren Q, Ma M et al. Comparison of ketamine, 7,8-dihydroxyflavone, and ANA-12 antidepressant effects in the social defeat stress model of depression. Psychopharmacology (Berl) 2015; 232: 4325-4335.

34 Yang B, Zhang JC, Han M, Yao W, Yang C, Ren Q et al. Comparison of R-ketamine and rapastinel antidepressant effects in the social defeat stress model of depression. Psychopharmacology (Berl) 2016; 233: 3647-3657.

35 Yang C, Qu Y, Abe M, Nozawa D, Chaki S, Hashimoto K. (R)-ketamine shows greater potency and longer lasting antidepressant effects than its metabolite (2R,6R)-hydroxynorketamine. Biol Psychiatry 2017; doi: 10.1016/j.biopsych.2016. 12.020 .
36 Ren Q, Ma M, Ishima T, Morisseau C, Yang J, Wagner KM et al. Gene deficiency and pharmacological inhibition of soluble epoxide hydrolase confers resilience to repeated social defeat stress. Proc Natl Acad Sci USA 2016; 113: E1944-E1952.

37 Yao W, Zhang JC, Ishima T, Dong C, Yang C, Ren Q et al. Role of Keap1-Nrf2 signaling in depression and dietary intake of glucoraphanin confers stress resilience in mice. Sci Rep 2016; 6: 30659.

38 Ma M, Ren Q, Yang C, Zhang JC, Yao W, Dong C et al. Adjunctive treatment of brexpiprazole with fluoxetine shows a rapid antidepressant effect in social defeat stress model: role of BDNF-TrkB signaling. Sci Rep 2016; 6: 39209.

39 Ren Q, Ma M, Yang C, Zhang JC, Yao W, Hashimoto K. BDNF-TrkB signaling in the nucleus accumbens shell of mice has key role in methamphetamine withdrawal symptoms. Transl Psychiatry 2015; 5: e666.

40 Zhang JC, Yao W, Dong C, Yang C, Ren Q, Ma M et al. Prophylactic effects of sulforaphane on depression-like behavior and dendritic changes in mice after inflammation. J Nutr Biochem 2017; 39: 134-144.

41 Zhang JC, Wu J, Fujita Y, Yao W, Ren Q, Yang C et al. Antidepressant effects of TrkB ligands on depression-like behavior and dendritic changes in mice after inflammation. Int J Neuropsychopharmacol 2015; 18: pyu077.

42 Yang C, Shirayama Y, Zhang JC, Ren Q, Yao W, Ma M et al. R-ketamine: a rapidonset and sustained antidepressant without psychotomimetic side effects. Transl Psychiatry 2015; 5: e632.

43 Yang C, Shirayama Y, Zhang JC, Ren Q, Hashimoto K. Regional differences in brainderived neurotrophic factor and dendritic spine density confer resilience to inescapable stress. Int J Neuropsychopharmacol 2015; 18: pyu121.

44 Schloss PD, Westcott SL, Ryabin T, Hall JR, Hartmann M, Hollister EB et al. Introducing mothur: open-source, platform-independent, community-supported software for describing and comparing microbial communities. Appl Environ Microbiol 2009; 75: 7537-7541.

45 Li W, Fu L, Niu B, Wu S, Wooley J. Ultrafast clustering algorithms for metagenomic sequence analysis. Brief Bioinform 2012; 13: 656-668.

46 Wang Q, Garrity GM, Tiedje JM, Cole JR. Naive Bayesian classifier for rapid assignment of rRNA sequences into the new bacterial taxonomy. Appl Environ Microbiol 2007; 73: 5261-5267.

47 McEwen BS. Physiology and neurobiology of stress and adaptation: central role of the brain. Physiol Rev 2007; 87: 873-904.

48 Duman RS, Aghajanian GK. Synaptic dysfunction in depression: potential therapeutic targets. Science 2012; 338: 68-72.

49 Ohgi Y, Futamura T, Hashimoto K. Glutamate signaling in synaptogenesis and NMDA receptors as potential therapeutic targets for psychiatric disorders. Curr Mol Med 2015; 15: 206-221.

50 Tanaka T, Narazaki M, Kishimoto T. Immunotherapeutic implications of IL-6 blockade for cytokine storm. Immunotherapy 2016; 8: 959-970.

51 Chourbaji S, Urani A, Inta I, Sanchis-Segura C, Brandwein C, Zink M et al. IL-6 knock-out mice exhibit resilience to stress-induced development of depressionlike behaviors. Neurobiol Dis 2006; 23: 587-594.

52 Yang C, Fujita Y, Ren Q, Ma M, Dong C, Hashimoto K. Bifidobacterium in the gut microbiota confer resilience to chronic social defeat stress in mice. Sci Rep 2017; 7: 45942.

53 Turnbaugh PJ, Ley RE, Mahowald MA, Magrini V, Mardis ER, Gordon Jl. An obesityassociated gut microbiome with increased capacity for energy harvest. Nature 2006; 444: 1027-1031.

54 Ley RE, Turnbaugh P, Klein S, Gordon Jl. Microbial ecology: human gut microbes associated with obesity. Nature 2006; 444: 1022-1023.

55 Jeffery IB, O'Toole PW, Öhman L, Claesson MJ, Deane J, Quigley EM et al. An irritable bowel syndrome subtype defined by species-specific alterations in faecal microbiota. Gut 2012; 61: 997-1006.

56 Mariat D, Firmesse O, Levenez F, Guimarăes V, Sokol H, Doré J et al. The Firmicutes/ Bacteroidetes ratio of the human microbiota changes with age. BMC Microbiol 2009; 9: 123.

57 Cho SY, Kim J, Lee JH, Sim JH, Cho DH, Bae IH et al. Modulation of gut microbiota and delayed immunosenescence as a result of syringaresinol consumption in middle-aged mice. Sci Rep 2016; 6: 39206.

58 Kamada N, Seo SY, Chen GY, Núñez G. Role of the gut microbiota in immunity and inflammatory disease. Nat Rev Immunol 2013; 13: 321-335.

59 Wang L, Christophersen CT, Sorich MJ, Gerber JP, Angley MT, Conlon MA Increased abundance of Sutterella spp. and Ruminococcus torques in feces of children with autism spectrum disorder. Mol Autism 2013; 4: 42.

60 Gophna U, Konikoff T, Bjørn Nielsen H. Oscillospira and related bacteria - from metagenomics species to metabolic features. Environ Microbiol 2017; 19: 835-841.

61 Eeckhaut V, Machiels K, Perrier C, Romero C, Maes S, Flahou B et al. Butyricicoccus pullicaecorum in inflammatory bowel disease. Gut 2013; 62: 1745-1752.

62 Devriese S, Eeckhaut V, Geirnaert A, Van den Bossche L, Hindryckx P, Van de Wiele $T$ et al. Reduced mucosa-associated Butyricicoccus activity in patients with ulcerative colitis correlates with aberrant claudin-1 expression. J Crohns Colitis 2017; 11: 229-236. 
63 Walters WA, Xu Z, Knight R. Meta-analyses of human gut microbes associated with obesity and IBD. FEBS Lett 2014; 588: 4223-4233.

64 Konikoff T, Gophna U. Oscillospira: a central, enigmatic component of the human gut microbiota. Trends Microbiol 2016; 24: 523-524.

65 Sun J, Wang F, Hong G, Pang M, Xu H, Li H et al. Antidepressant-like effects of sodium butyrate and its possible mechanisms of action in mice exposed to chronic unpredictable mild stress. Neurosci Lett 2016; 618: 159-166.

66 Wang AK, Miller BJ. Meta-analysis of cerebrospinal fluid cytokine and tryptophan catabolite alterations in psychiatric patients: comparisons between schizophrenia, bipolar disorder, and depression. Schizophr Bull 2017; doi: 10.1093/schbul/sbx035.
This work is licensed under a Creative Commons Attribution 4.0 International License. The images or other third party material in this article are included in the article's Creative Commons license, unless indicated otherwise in the credit line; if the material is not included under the Creative Commons license, users will need to obtain permission from the license holder to reproduce the material. To view a copy of this license, visit http://creativecommons.org/licenses/ by/4.0/

(c) The Author(s) 2017 